\title{
The Parameters Selection of PSO Algorithm influencing On performance of Fault Diagnosis
}

\author{
Yan $\mathrm{HE}^{1, \mathrm{a}}$, Wei Jin MA ${ }^{1}$ and Ji Ping ZHANG ${ }^{1}$ \\ ${ }^{1}$ School of Mechanical Engineering and Power Engineer, North University of China, Taiyuan, Shanxi 030051, China
}

\begin{abstract}
The particle swarm optimization (PSO) is an optimization algorithm based on intelligent optimization. Parameters selection of PSO will play an important role in performance and efficiency of the algorithm. In this paper, the performance of PSO is analyzed when the control parameters vary, including particle number, accelerate constant, inertia weight and maximum limited velocity. And then PSO with dynamic parameters has been applied on the neural network training for gearbox fault diagnosis, the results with different parameters of PSO are compared and analyzed. At last some suggestions for parameters selection are proposed to improve the performance of PSO.
\end{abstract}

\section{Introduction}

Particle swarm optimization (PSO) is an optimization algorithm which is based on swarm intelligent[1]. The PSO algorithm is initialized with a population of random candidate solution. Swarm intelligent produced by cooperation and competition of particles between population, guides optimization and search. PSO has much more profound intelligent background and could be performed easily and simply. Based on its advantages, the PSO has been applied in function optimization, artificial neural network training, fuzzy control system and some other fields at the present .

Parameters selection of PSO is the key influence on performance and efficiency of the algorithm. It is a complicated optimized problem that how to determine the optimum parameters for the optimal performance. There are no general methods to determine the optimum parameters, which are selected by user experience, because there are different parameter spaces between different parameters and relativity each other[2]. However, the regularity of different parameter which influences the performance of algorithm may be found. In this paper, the performance of PSO is analyzed, based on the control parameters variants including particle number $m$, accelerate constant $c_{1}$ and $c_{2}$, inertia weight $w$ and maximum limited velocity $v_{\max }$. And then the effect of these parameters of PSO is applied in the neural network training for gearbox, the fault diagnosis results with different parameters of PSO are compared and analyzed. At last some suggestions for parameters selection are proposed. .

\section{Particle Swarm Optimization Algorithm}

Particle swarm optimization algorithm is a method which makes each particle in the population follow the current superior particle by the certain speed, searches problems' optimal solution in the solution space.

Particle flying speed is adjusted dynamically by individual and community's flight experience[3].The particle swarm algorithm formula as follows:

$$
\begin{gathered}
v_{i d}(t+1)=w v_{i d}(t)+c_{1} r_{1}\left(p_{i d}-x_{i d}(t)\right)+c_{2} r_{2}\left(p_{g d}-x_{i d}(t)\right) \\
x_{i d}(t+1)=x_{i d}(t)+v_{i d}(t+1)
\end{gathered}
$$

where $x_{i d}(t)$ is the current position of particle $i$, $p_{i d}$ is superior position of particle $i$, which searches until now, $p_{g d}$ is the entire particle swarm' $\mathrm{s}$ superior position. $w$ is positive constants, called inertia factor which is linearly reducing with iteration; $c_{1}$ and $c_{2}$ are non- negative constants, called cognitive learning rate. $r_{1}$ and $r_{2}$ are random numbers in range [0 $1] ; v_{i d} \in\left[-v_{\max }, v_{\max }\right] ; v_{\max }$ is non-negative constant. The selected condition of stopping iteration is the max iteration number or the best position of particle searched till now meets for adaptive thresholds significance.

In formula (1), the velocity change of PSO includes three components: the momentum components, the cognitive components and the social components. It determines the performances of PSO how to balance

\footnotetext{
a Yan HE:zbhy91@163.com
} 
three components. This evolution equation has been used as a standard PSO algorithm.

\section{Parameters Selection}

The PSO algorithm includes some tuning parameters, such as inertia weight $w$, particle number $m$, accelerate constant $c_{1}$ and $c_{2}$, maximum limited velocity $v_{\max }$, the max iteration number $T_{\max }$, and computed precision $\varepsilon$.Of them, $w, m, c_{1}, c_{2}$ and $v_{\max }$ are used as mainly controlling parameters, at the same time $T_{\max }$ and $\varepsilon$ are used as the conditions of stopping iteration.

\subsection{Analysis of inertia weight selection}

Inertia weight describes that the previous velocity influence on current velocity. Controlling its selection may tune the global and local search ability of PSO. If $w=0$ the velocity of particle depends on its current position pbest ( the personal best ) and gbest (the global best). The velocity has no memory. If $w \neq 0$, the particle has the tendency of exploring new space, and the larger is the $w$, the larger is the flying velocity of the particle, at the same time the particle will explore with longer steps. The smaller is the $w$, the smaller is the flying velocity of the particle, and then the particle will tend to dedicate local exploitation.

Presently, the inertia weight used more is linearly decreasing weight (simply called LDW), which is proposed by Shi, as following:

$w=w_{\max }-\frac{w_{\max }-w_{\min }}{T_{\max }} t$

Where $w_{\max }$ is the maximum inertia weight, and $w_{\min }$ is the minimum inertia weight, typically $w_{\max }=0.9, \quad w_{\min }=0.4 ; \quad t$ is current iteration number; $T_{\max }$ is the maximum iteration number.

\subsection{Analysis of accelerate constant selection}

Accelerate constant $c_{1}$ and $c_{2}$ represent the particle stochastic acceleration weight toward the personal best (pbest) and the global best (gbest). Small accelerate constant may induce the particle wandering away in goal area; however, large accelerate constant may induce that the particle move quickly to the goal area, even fly away from it.If $c_{1}=c_{2}=0$, the particle will fly with current velocity till the border. At that moment, it is difficult for the particle to find the good solution, because it has to search in definite area. When $c_{1}=0$, the particle has no the cognitive ability, which is social-only, and the algorithm has the ability to get to the new searching area by particles' cooperation for each other. Its convergent speed is faster than standard algorithm. But encountering complicate problem, it is easily getting into local optima.
When $c_{2}=0$, there isn't shared information between particles, which is cognition-only, so a swarm with particle number $m$ equals to $m$ single particle. The probability to get the optima is very small because individual has no interaction.

In previous researches, Kennedy and Eberhart describe that a relatively high value of the cognitive component, compared with the social component, will result in excessive wandering of the search space[1]. In contrast, a relatively high value of the social component may lead particles to rush prematurely toward local optimum. Moreover, they suggested setting either of the acceleration coefficients at 2 in order to make the mean of both stochastic factors in (1) unity. Later, Clerc deduced $c_{1}=c_{2}=2.05$, some other researchers thought that $c_{1}$ did not equal $c_{2}$, and reached a conclusion $c_{1}=2.8$ from experiments. In reality, these researches have been constricted to the application of part problems. So it had not popularized all fields. Suganthan tested a method of linearly decreasing both acceleration coefficients with time, but observed that fixed acceleration coefficients at 2 generate better solutions [4]. However, through empirical studies he suggested that the acceleration coefficients should not be equal to 2 all the time[5].

Generally, in population-based optimization methods, it is described to encourage the individuals to wander through the entire search space, without cluster around local optima, during the latter stages, it is very important to enhance convergence toward the global optima, to find the optimum solution efficiently.

\subsection{Analysis of particle number of population selection}

Shi and Eberhart found that PSO was not sensitive to particle number[6]. Their conclusion was based on the mean value of needed iteration under given precision. Zhang Li-ping has researched the mean values of all kinds of function with population variant, such as Sphere ,De Jong' s f4, Rosenbrock, Rastrigin ,Griewank Shaffer' sf6 Ackley[7]. The conclusion was that their effect on PSO was smaller, when particle numbers were more than 50; which were the same as the conclusion of Shi and Eberhart. However, when particle numbers were less than 50, they greatly influenced on the performance of PSO. From the computed complexity analysis, more particles require more function evaluation, thereby increase more computing time, and increase the reliability simultaneously.

\subsection{Analysis of the max velocity selection}

The velocity of particle is usually constricted to a range in order to decrease the probability of particle moving away from searching space in evolutionary process. $v_{\max }$ is the maximum limited velocity of a particle. If $v_{\text {max }}$ is excessively large, it is thought that the velocity is not limited, then the particle may fly through the good 
solution. At that moment, the performance of PSO depends on inertia weight; in the contrast, If $v_{\text {max }}$ is excessively small, the particle may not explore the space outer local best area. So it easily gets into local best. For these reasons, the excessively big or small velocity may lead the performance of algorithm decreasing.

\section{The Application Of PSO Algorithm On Neural Network}

\subsection{Structure of neural network for gearbox fault diagnosis}

In this diagnostic system, the gearbox is a researched object. Because gearbox is a very complicated transfer mechanism, its relations with faults and symptoms are not very definite and of nonlinear map, while BP neural network models have the properties such as good self-learning, self-adapting, associative memory and identification of nonlinear model, and particularly suitable for complicated pattern identification, so they are widely used in fault diagnosis of gear.

Here, the gearbox of a tractor is the researched object, and some feature parameters of frequency-domain are selected, based on gear fault. So gearbox fault diagnosis system is defined by three-layer BP neural network with topology architecture 15-31-3. The outputs of neural network are relative to fault types. There are 3 fault types, which are expressed no faults, root of tooth crack, and gear tooth collapsing.

\subsection{The analysis of parameters selection of PSO influence on performance of fault diagnosis.}

The simulation experiments were performed in neural network with standard PSO, when the main control parameters were tuned including particle number $m$ and maximum limited velocity $v_{\max }$. Then their effect on neural network training process and fault diagnosis results has been researched and analyzed.

\subsubsection{The effect of different particle number}

In simulation experiments, on the conditions including given $\varepsilon=0.001, V_{\max }=1$ and $T_{\max }=2000$, particle numbers $m$ was changed, and 9 group samples of gearbox were inputted neural network to train it, the training error cures of network with different particle numbers have been showed in Figure.1(a) and (b).

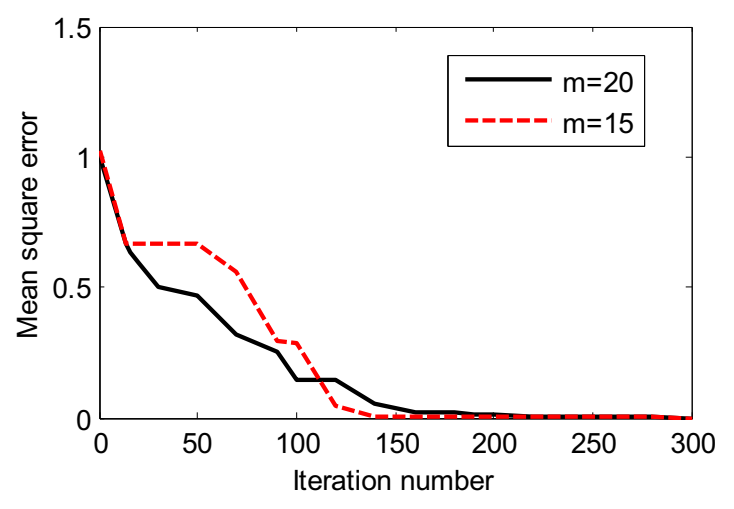

(a) $m=15, \quad m=20$

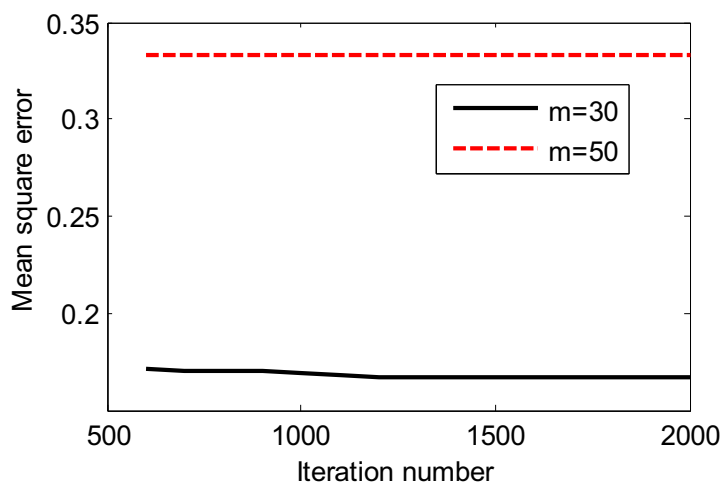

(b) $m=30, \quad m=50$

Figure 1. The training error cure of network with different $m$

When $m$ is 15 and 20 , the error cures convergent speedily, observed from Figure.1 (a); and iteration number being about 150 , the mean square errors have reached specified precision 0.001 . When $m$ is 30 and 50 , the error cures convergent slowly, observed from Fig.1(b); and iteration number being the maximum 2000, the individual mean square error is 0.1668 and 0.3333 , which has not reached specified precision 0.001 , and has got stuck in local best .

\subsubsection{The effect of the max velocity}

In previous researches, usually $v_{\max }$ was constant 1 .In this study, the performance of PSO with changing $v_{\max }$ is observed. As formula (4) shows, $v_{\max }$ is changed with iteration number, and the PSO with dynamic $v_{\max }$ is called VPSO.

$$
\mathrm{v}_{\text {max }}=\left(\mathrm{e}^{1-\mathrm{t} / \mathrm{T}_{\max }}\right) \mathrm{V}_{\mathrm{c}}
$$

Where $v_{\text {max }}$ is the variable maximum which limited velocity, $\mathrm{t}$ is current iteration number, $T_{\max }$ is the maximum iteration number, and $V_{c}$ is set velocity constant.

This algorithm was applied to simulation experiments in neural network with 15-31-3 topology architecture. Of them the other parameters were set as follow: 
$m=20$,fitness threshold $\varepsilon=0.001, V_{c}=1.5$,

$T_{\max }=2000.9$ group samples of gearbox were inputted neural network to train it, showed by Table1,ideal output type is set three types such as no faults ( $\left(\begin{array}{lll}1 & 0 & 0\end{array}\right)$,root of tooth crack $\left(\begin{array}{lll}0 & 1 & 0\end{array}\right)$ and gear tooth collapsing $\left(\begin{array}{lll}0 & 0 & 1\end{array}\right)$. The training error cures of network by PSO and VPSO have been showed in Figure 2. The error cure by VPSO convergent speedily observed from it. When iteration number reached 200, its error has satisfied a required accuracy target, which is fitting threshold $\varepsilon=0.001$.

Table 1. Feather samples of Gearbox

\begin{tabular}{|c|c|c|}
\hline $\begin{array}{c}\text { Data } \\
\text { number }\end{array}$ & Feature sample & $\begin{array}{l}\text { Fault } \\
\text { type }\end{array}$ \\
\hline 1 & $\begin{array}{lllllll}0.2286 & 0.1292 & 0.0072 & 0.1592 & 0.1335 \\
0.0733 & 0.1159 & 0.0940 & 0.0522 & 0.1345 \\
0.0090 & 0.1260 & 0.3619 & 0.0690 & 0.1828\end{array}$ & 1. \\
\hline 2 & $\begin{array}{lllll}0.2090 & 0.0947 & 0.1393 & 0.1387 & 0.2558 \\
0.0900 & 0.0771 & 0.0882 & 0.0393 & 0.1430 \\
0.0126 & 0.1670 & 0.2450 & 0.0508 & 0.1328 \\
\end{array}$ & 1 \\
\hline 3 & $\begin{array}{lllllll}0.0442 & 0.0880 & 0.1147 & 0.0563 & 0.3347 \\
0.1150 & 0.1453 & 0.0429 & 0.1818 & 0.0378 \\
0.0092 & 0.2251 & 0.1516 & 0.0858 & 0.0670\end{array}$ & 1 \\
\hline 4 & $\begin{array}{lllll}0.2603 & 0.1715 & 0.0702 & 0.2711 & 0.1491 \\
0.1330 & 0.0968 & 0.1911 & 0.2545 & 0.0871 \\
0.0060 & 0.1793 & 0.1002 & 0.0789 & 0.0909\end{array}$ & 2 \\
\hline 5 & $\begin{array}{llllll}0.3690 & 0.2222 & 0.0562 & 0.5157 & 0.1872 \\
0.1614 & 0.1425 & 0.1506 & 0.1310 & 0.0500 \\
0.0078 & 0.0348 & 0.0451 & 0.0707 & 0.0880 \\
\end{array}$ & 2 \\
\hline 6 & $\begin{array}{lllll}0.0359 & 0.1149 & 0.1230 & 0.5460 & 0.1977 \\
0.1248 & 0.0624 & 0.0832 & 0.1640 & 0.1002 \\
0.0059 & 0.1503 & 0.1837 & 0.1295 & 0.0700\end{array}$ & 2 \\
\hline 7 & $\begin{array}{llllll}0.1759 & 0.2347 & 0.1829 & 0.1811 & 0.2922 \\
0.0655 & 0.0774 & 0.2273 & 0.2056 & 0.0925 \\
0.0078 & 0.1852 & 0.3501 & 0.1680 & 0.2668 \\
\end{array}$ & 3 \\
\hline 8 & $\begin{array}{llllll}0.0724 & 0.1909 & 0.1340 & 0.2409 & 0.2842 \\
0.0450 & 0.0824 & 0.1064 & 0.1909 & 0.1586 \\
0.0116 & 0.1698 & 0.3644 & 0.2718 & 0.2494\end{array}$ & 3 \\
\hline 9 & $\begin{array}{llllll}0.2634 & 0.2258 & 0.1165 & 0.1154 & 0.1074 \\
0.0657 & 0.0610 & 0.2623 & 0.2588 & 0.1155 \\
0.0050 & 0.0978 & 0.1511 & 0.2273 & 0.3220 \\
\end{array}$ & 3 \\
\hline
\end{tabular}

Note:Fault type: 1:no faults ,2:Root of tooth crack,3:Gear tooth collapsing

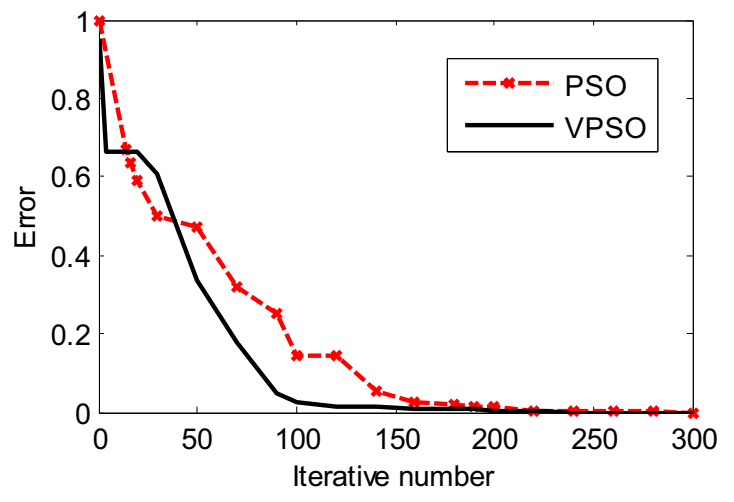

Figure 2. The training error cure of network based on

\section{VPSO and PSO}

Figure 2 shows the training error cures based on VPSO and PSO. To the same group samples, two algorithms convergent quickly under 50 steps; but evolution steps being between 50 and 100, VPSO obviously convergent more quickly than PSO. It is indicated that, $v_{\max }$ linearly varying with iteration number, plays the role of accelerating convergent speed.

Two output mean error cures have been showed in Figure 3(a) and (b) ,when fault types of gearbox are root of tooth crack and gear tooth collapsing. The mean errors with VPSO are obviously smaller compared with PSO. It indicates that performance of algorithm is better, and the accuracy and efficient of fault diagnosis are obviously improved, when dynamic $v_{\max }$ is following with iteration number.

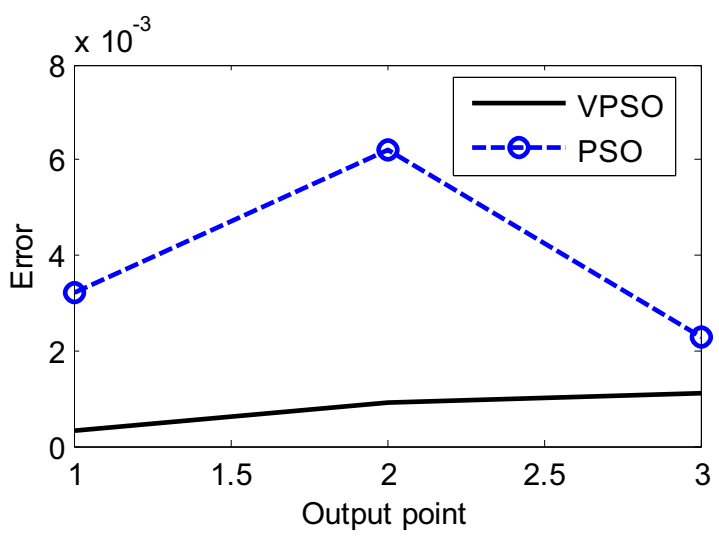

(a) Root of tooth crack.

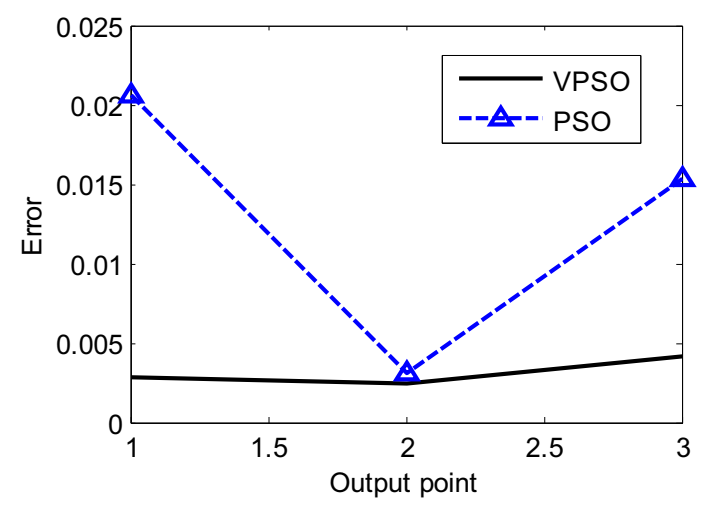

(b) Gear tooth collapsing

Figure 3. The output mean error cure in different fault

\section{Conclusion}

In this paper the fault diagnosis results with different parameters of PSO have been compared and analyzed. The conclusion is as follows:

(1) As particle number $m<100$, the effect of $m$ variant on the training performance of networks and diagnostic accuracy, is obvious; As $m>100$, the effect on them is not obvious. With particle number increasing, 
the reliability of PSO algorithm improved, but more particles require more functional evaluation, so the required time of the algorithm increases, the efficient is poor.

Therefore, selection particle number should base on architecture of networks, and consider the reliability of the algorithm and the required time. Generally speaking, the particle number is 20 enough to usual problem, but the particle number may be selected 50 to some complicate problem.

(2) The performance of algorithm is better, and the accuracy and efficient of fault diagnosis are obviously improved, and the convergent speed is accelerating when the maximum velocity $v_{\max }$ is set a dynamic parameters following with iteration number. The $v_{\max }$ indirectly influences the global searching ability. Dynamic modification $v_{\max }$ may induce particle which always searches in rational space in order to prevent particle wandering through the searching space or toward the local optima.

\section{Acknowledgment}

The authors acknowledge the financial support of the National Nature Science Foundation of Shanxi Province contract/grant number: 2015011063.

\section{References}

1. J .Kennedy and R. C. Eberhart,Particle swarm optimization, Proc. IEEE Int.Conf. Neural network, pp.1942-1948,(1995)

2. Jian-chao Zeng, Jing Jie, Zhi-hua Cui, Particle swarm optimization algorithm, Sience publication, (2004)

3. Ioan Cristian Trelea, The particle swarm optimization algorithm: convergence analysis and parameter selection, Information Processing Letters, 85, pp.317-325, (2003).

4. P.N. Suganthan, Particle swarm optimization with neighborhood operator, Proc. IEEE Int.Conf. Evolution computation ,3, pp.1958-1962,( 1999),

5. Asange Ratnawwra, Saman K.Halgamuge, Harry C.Waston, Self-organizing hierarchical particle swarm optimizer with time-vary acceleration Coefficients, IEEE Transaction on evolutionary computation, 8, pp.240-255, 3(2004)

6. Y.Shi, R.C.Ebethart, A modified particle swam optimizer, Proc. IEEE International Conference on Evolutionary Computation, pp. 69-73,( 1998)

7. Li-ping Zhang, The Theorem and Practice upon the Particle Swarm Optimization Algorithm. Zhejiang University, Dissertation Submitted to Zhejiang University for the Degree of Doctor of Philosophy, (2005) 